\title{
The Effects of Redundancy in Multiple-Cue Probability Learning
}

Michael K. Lindell and Thomas R. Stewart

Institute of Behavioral Science, University of Colorado

The 80 subjects had a task of a two-cue probability lcarning at a $+.8,+.4, .0$, -.4 , or -.8 level of stimulus redundancy. An analysis of 50 lcarning trials revealed a significant main effect of redundancy. Performance was higher when redundancy was lower. These findings are interpreted in terms of task characteristics that accompany redundancy, and the implications for the learning of complex inference tasks are discussed.

In their study of the role of redundant information in inference tasks, Knowles, Hammond, Stewart, and Summers (1971) found the rate of learning to be influenced by both the sign (positive or negative) and the magnitude of the intercorrelation of two cues. Their finding that learning varies with cue intercorrelation, in the order $+.8>+.4>-.4>-.8$, is not conclusive, however, because of the inevitable confounding of certain parameters of the multiple-cue training task with redundancy. Specifically, Knowles et al. used four training tasks with four different levels of cue intercorrelation (redundancy). They fixed the ratio of the regression weights $\left(b_{e i}\right)$ in each task at $2: 1$; the squared multiple correlation between the two cues and the criterion was set at .84. As they acknowledged, this design could be carried out only by letting cue validities (correlations between each cue and the criterion, $r_{e i}$ ) vary. Therefore, the four training tasks varied not only in redundancy but also in cue validities.

The purpose of the present study was to determine whether the results obtained by Knowles et al. were a result of varying levels of redundancy or varying levels of other task parameters. This was accomplished by using the same levels of redundancy as in the earlier study but fixing the ratio of cue validities across tasks rather than fixing the ratio of regression weights. The hypothesis was that the results of Knowles et al. on the effect of redundancy would not generalize to these conditions. Confirmation of this hypothesis would indicate that task parameters other than redundancy are important in influencing the rate of learning. 


\section{METHOD}

The subjects were 80 introductory psychology students, who took part in the experiment to satisfy a course requirement. They were randomly assigned to one of the five tasks (intercorrelations of $+.8,+.4, .0,-.4$, or -.8 ), 16 subjects per task.

With the exception of the different statistical relations, the task was the same as that of Knowles et al. - a simulated medical diagnosis in which the subjects were instructed to estimate 'virus level' from observation of the values of two fictitious laboratory tests, 'Rel. B.V.' and 'Niprog.'

Table 1 presents the parameters for the five tasks. All redundancy levels included in the Knowles et al. study were also included in this experiment. The differences in other parameters are due to the fact that the ratio of cue validities was fixed in the present study, while Knowles et al. fixed the ratio of the regression weights.

\section{RESULTS}

The measure of performance, referred to here as $r_{p}$, is the correlation between the subjects' judgments and the linearly predictable portion of the criterion. ${ }^{1}$ This measure, after transformation to Fisher's $z$ score, was analyzed in a 5 (tasks, i.e., intercorrelations) $\times 5$ (blocks) ANOVA with blocks treated as a repeated measure. There were significant effects of task $(+.4>0>-.4>+.8>-.8)$, of blocks (improvement over blocks), and of the interaction of task and blocks (see Table 2). The inter-

Table 1. Task characteristics

\begin{tabular}{rrrrrrrr}
\hline Task & $r_{e 1}$ & $b_{e 1}$ & $r_{e 2}$ & $b_{e 2}$ & $R_{e}{ }^{2}$ & $r_{e 1} / r_{e 2}$ & $b_{e 1} / b_{e 2}$ \\
\hline+.8 & .410 & $(-.684)$ & .820 & $(1.367)$ & .84 & .50 & -.50 \\
+.4 & .455 & $(.109)$ & .910 & $(.865)$ & .84 & .50 & .13 \\
.0 & .410 & $(.410)$ & .820 & $(.820)$ & .84 & .50 & .50 \\
-.4 & -.455 & $(-.109)$ & .910 & $(.865)$ & .84 & -.50 & .13 \\
-.8 & -.410 & $(.683)$ & .820 & $(1.367)$ & .84 & -.50 & .50 \\
\hline
\end{tabular}

Table 2. ANOVA summary

\begin{tabular}{lcc}
\hline & \multicolumn{2}{c}{$r_{p}$} \\
\cline { 2 - 3 } & $F$ & $\begin{array}{c}\text { Level of } \\
\text { significance }\end{array}$ \\
\hline Task & 4.73 & $<.01$ \\
Blocks & 9.21 & $<.001$ \\
Task $\times$ Blocks & 2.06 & $<.025$ \\
\hline
\end{tabular}

action was due primarily to the fluctuations with the +.8 and -.8 tasks and to the greater improvement in performance with the +.8 and -.4 tasks (see Figure 1).

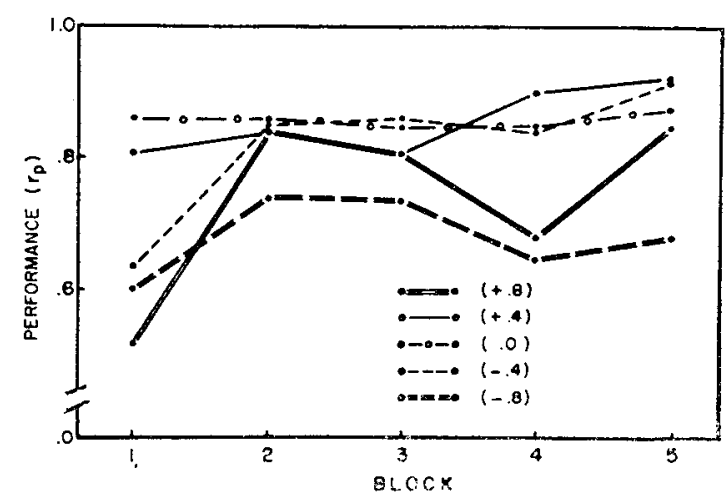

Figure 1. Mean performance $\left(r_{p}\right)$ as a function of blocks of ten trials for subjects in five intercorrelation conditions

These results can be summarized as follows. First, the finding by Knowles et al. that performance does not improve as a direct function of the magnitude of redundancy was clearly replicated. However, in contrast to the previous study, where the tasks were ordered $+.8>+.4$ $>-.4>-.8$ with respect to performance, no systematic relationship between the sign of the intercorrelation and performance was observed. Last, the variation in performance cannot be explained simply by the variation in redundancy alone, or by the variation in ratio of the cue weights alone, or by the variation in cue validities alone - all three parameters affect performance.

\section{DISCUSSION}

The difficulty of multiple-cue probability learning is clearly a function of more than one task parameter. The determination of exactly how combinations of task parameters interact to produce tasks of varying levels of difficulty is a problem for a program of research involving a wide variety of tasks. The research to date suggests that two properties have an important influence on performance. The properties, each of which is dependent on a combination of statistical task parameters, are task sensitivity and congruity of task parameters. Each is discussed below. 
Task sensitivity refers to the degree to which performance suffers as the subject's weights $\left(b_{s i}\right)$ differ from the optimal, task, regression weights $\left(b_{e i}\right)$. For example, if the correlation between the cues approaches 1.0, then the task will be insensitive and the subject's performance would suffer little even if his weights were quite different from the task weights. He should achieve high performance in such a task unless he is inconsistent or uses weights opposite in sign to the correct weights. Tasks with lower correlations among the cues may be more sensitive and less forgiving of deviations from the optimal weights. Performance would of course be lower on such tasks.

Task sensitivity, however, is not determined solely by cue intercorrelation. For example, Knowles et al. used a task that had a cue intercorrelation of +.8 and was very insensitive, while the task that had a +.8 cue intercorrelation in the present study was quite sensitive. Sensitivity depends on the combination of cue intercorrelations, weights, and multiple correlation present in the task.

Because tasks vary in sensitivity, performance ( $r_{p}$, or any other 'global' correlational measure) is not always a valid indicator of how well the parameters of a subject's system match the parameters of the task system. This is illustrated by comparing Figures 2 and 3 with Figure 1. Figures 2 and 3 show how well the subjects' regression weights $\left(b_{s i}\right)$ matched the optimal task weights. It is easy to see that the pattern of weight matching differs substantially from the pattern of performance. High performance is not always an indication that a subject has learned the correct task parameters.

Congruity of task parameters refers to the similarity between cue validities $\left(r_{e i}\right)$ and the corresponding beta weights for standardized scores $\left(\beta_{e i}\right)$. When cues are orthogonal, cue validities and beta weights are identical and congruence is perfect. As cue intercorrelations differ from zcro, discrepancics between cue validities and beta weights are possible. $\Lambda$ a result, cue-criteria correlations $\left(r_{e i}\right)$ no longer indicate the appropriate weights $\left(\beta_{e i}\right)$ for prediction. These discrepancies can be particularly important when $r_{e i}$ and $\beta_{e i}$ are of opposite sign for one or more cues - when the task contains a suppressor variable (see Lord and Novick, 1968 , p. 272). Such was the case for cue 1 in the +.8 and -.8 tasks in the present study. A naive subject unaided by statistical computations will have considerable difficulty in diagnosing the existence and function of a suppressor variable in a task. Failure to properly use the suppressor variable will result in a performance decrement (see Figure 3 ).

In sum, the results of the present study suggest that there is no simple effect of cue intercorrelation on multiple-cue probability learning. Rather,

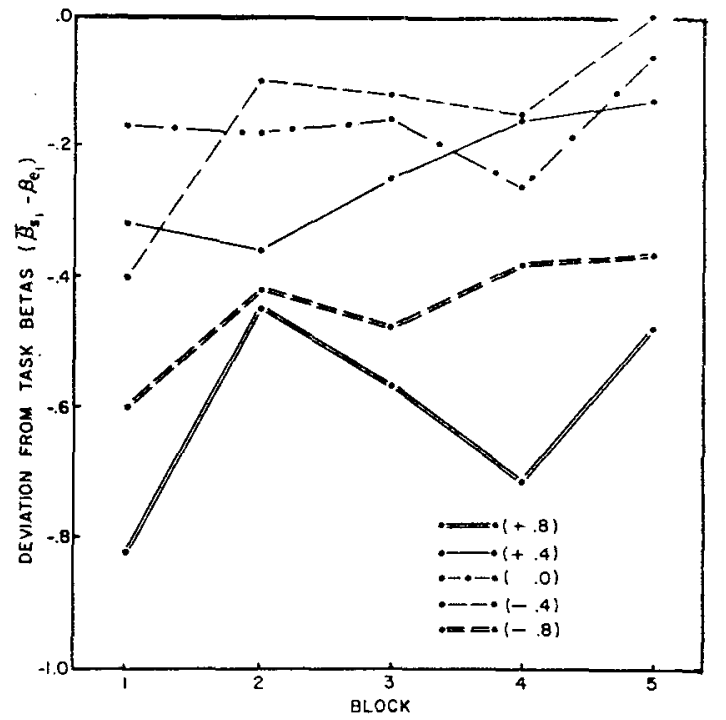

Figure 2. Mean deviation from task regression weights for cue $1\left(\bar{\beta}_{s_{1}}-\beta_{e_{1}}\right)$

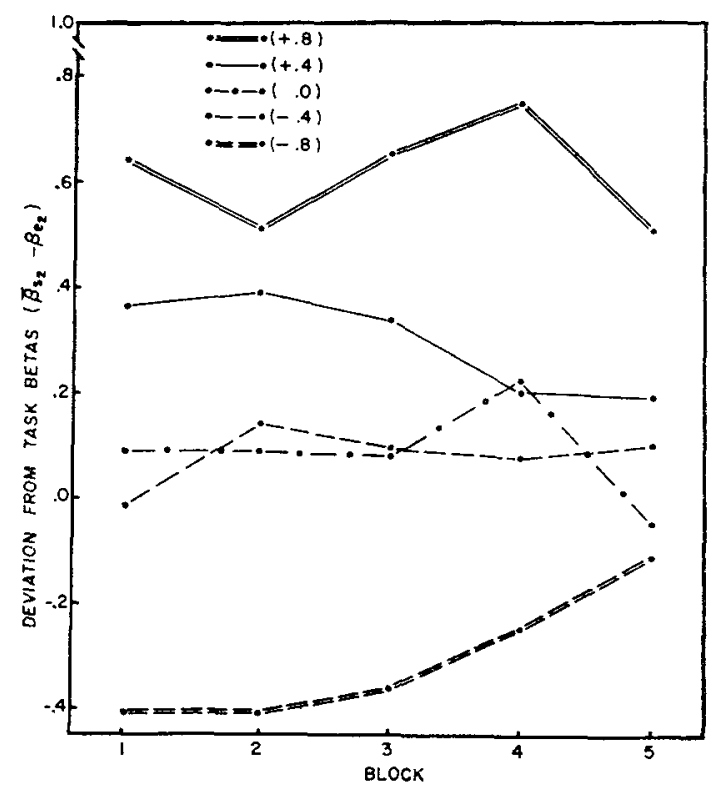

Figure 3. Mean deviation from task regression weights for cue $2\left(\bar{\beta}_{s_{2}}-\beta_{e_{2}}\right)$ 
the cue intercorrelation and the weights of the cues $\left(\beta_{e i}\right)$ jointly have two complex effects, task sensitivity and congruity of task parameters, both of which affect learning. A fuller understanding of multiple-cue probability learning awaits further research on these methodological considerations.

\section{Notes}

This research was supported by National Institute of Mental Health Grant MH16437. The authors wish to thank Kenneth $R$. Hammond for his contributions to this paper. Requests for reprints should be sent to Thomas R. Stewart, Institute of Behavioral Science, University of Colorado, Boulder, Colorado 80302. Revision received for publication April 11, 1973.

1. This measure was first used in a study of multiple-cue probability learning by Peterson, Hammond and Summers (1965) and was previously defined as $r_{c e}$ by Dudycha and Naylor (1966).

\section{References}

Dudycha, L. W., and Naylor, J. G. 1966. Characteristics of the human inference process in complex choice behavior situations. Organizational Behavior and Human Performance 1:110-128.

Knowles, B. A., Hammond, K. R., Stewart, T. R., and Summers, D. A. 1971. Positive and negative redundancy in multiple cue probability tasks. Journal of Experimental Psychology 90:157-159.

Lord, F. M., and Novick, M. R. 1968. Statistical theories of mental test scores. Reading, Mass.: Addison-Wesley.

Peterson, C. R., Hammond, K. R., and Summers, D. A. 1965. Optimal responding in multiple cue probability learning. Journal of Experimental Psychology $70: 270-276$. 